Please do not remove this page

RMIT

UNIVERSITY

\title{
Cognitive relay nodes for airborne LTE emergency networks
}

Hourani, Akram; Sithamparanathan, Kandeepan

https://researchrepository.rmit.edu.au/esploro/outputs/9921859627701341/filesAndLinks?institution=61RMIT_INST\&index=null

Hourani, A., \& Sithamparanathan, K. (2013). Cognitive relay nodes for airborne LTE emergency networks. Proceedings of the 7th International Conference on Signal Processing and Communication Systems (ICSPCS), 1-9. https://doi.org/10.1109/ICSPCS.2013.6723940

Document Version: Accepted Manuscript

Published Version: https://doi.org/10.1109/ICSPCS.2013.6723940

Repository homepage: https://researchrepository.rmit.edu.au

(C) 2013 IEEE

Downloaded On 2023/04/26 10:58:09 +1000

Please do not remove this page 
Thank you for downloading this document from the RMIT Research Repository.

The RMIT Research Repository is an open access database showcasing the research outputs of RMIT University researchers.

RMIT Research Repository http://researchbank.rmit.edu.au/

\section{Citation:}

Hourani, A and Sithamparanathan, K 2013, 'Cognitive relay nodes for airborne LTE emergency networks', in Tadeusz A Wysocki and Beata J Wysocki (ed.) Proceedings of the 7th International Conference on Signal Processing and Communication Systems (ICSPCS), United States, 16 - 18 December, 2013, pp. 1-9.

See this record in the RMIT Research Repository at:

https://researchbank.rmit.edu.au/view/rmit:23153

Version: Accepted Manuscript

Copyright Statement: (C) 2013 IEEE

Link to Published Version:

http://dx.doi.org/10.1109/ICSPCS.2013.6723940 


\title{
Cognitive Relay Nodes for
}

\section{Airborne LTE Emergency Networks}

\author{
Akram Al-Hourani, (Member IEEE) and Sithamparanathan Kandeepan (Senior Member IEEE) \\ School of Electrical and Computer Engineering, RMIT University, Melbourne, Australia. \\ Emails: akram.hourani@ieee.org, kandeepan@iiee..org
}

\begin{abstract}
This paper is proposing a novel concept of Cognitive Relay Node for intelligently improving the radio coverage of an airborne LTE emergency network, considering the scenarios outlined in the ABSOLUTE research project. The proposed network model was simulated comparing the different cases of deploying relay nodes to complement the coverage of an aerial LTE network. Simulation results of the proposed Cognitive Relay Nodes show significant performance improvement in terms of radio coverage quantified by the regional outage probability enhancement. Also, this paper is presenting the methodology and results of choosing the optimum aerial eNodeB altitude.
\end{abstract}

Index Terms-Cognitive Relay Node, LTE-A, Public Safety Network, Low Altitude Platform, Aerial Base Station.

\section{INTRODUCTION}

The unprecedented advancements in Long Term Evolution (LTE) System made it the practical choice for any -near future- public safety network deployment. By enabling multimedia contents and file sharing, a whole new dimension will be added to the public safety operations, significantly assisting critical tasks, and facilitating the sensitive time dependent missions of the dispatched resources in the field. Policemen could download suspects pictures, firemen can share the layout plan of a building, and ambulance vehicles could be fitted with live video stream connection to hospitals, allowing doctors to give lifesaving instructions. In fact LTE has been endorsed by US major public safety organizations as the technology of choice for the $700 \mathrm{MHz}$ band [4].

However, along with the projected increasing dependency on LTE System by public safety agencies, come new challenges facing network realization, mainly: (i) to provide continuous radio coverage in order to guarantee a seamless service during nominal operation conditions, and (ii) also it is very important to have a solid solution for a rapidly deployable network coverage as a failover scenario in emergency cases, i.e when extreme events occur such as natural disasters. Tackling the first challenge lays within the LTE-Advanced standardization itself that includes multiple enhancements over LTE Release 8 allowing cost effective deployment techniques, enabled by the Heterogeneous Network concept (HetNET), where several types of network elements can contribute in radio coverage such as Remote Radio Heads, Relay Nodes, Femto Cells and Pico Cells, thus pushing network infrastructure closer to end users for enhancing spectral efficiency. In fact some of those techniques are nowadays already in use by commercial network operators.

The serious challenge that still lacks standardization is in providing a rapidly deployable coverage alternative when a natural disaster occurs, during which, the performance of the main network is severely affected.

In this paper we are using the term nominal network referring to the conventional LTE network that is providing radio coverage during normal working conditions, while the term Rapid Emergency Network (REN); is used to refer an assumed rapidly deployable network infrastructure that enables basic telecom services such as voice and limited data, for a wide geographical afflicted area when the nominal network service has failed or severely affected by a mean of natural disaster; like flood, bushfire, severe storm or earthquake.

The REN architecture will be capable of serving sudden increases in mobile traffic caused by massive public events where vast population is expected in a certain area, e.g. sport events. The crucial role of REN in enabling public safety operations during the aftermath of disasters, has urged several governmental organizations to seek reliable deployment scenarios for such networks, for example the Public Safety and Homeland Security Bureau in the USA has endorsed the use of deployable aerial communications architecture (DACA) [3] that envisioned the recovery of critical communications for first responders within 12 to 18 hours. Another example of REN development efforts is the ongoing European Union funded ABSOLUTE project [2] (Aerial Base Stations with Opportunistic Links for Unexpected and Temporary Events) focusing on Low Altitude Platforms (LAP) that are increasingly believed as one of the ultimate answers for RENs.

Cognitive radio networks has been widely discussed for intelligently adapting the network, communications parameters and the available resources based on the radio environmental conditions $[14,15]$. In our work we consider such intelligence is available in the network, enabled by learning and sensing techniques [16-30], for intelligently making decisions based on the network and the radio environmental conditions.

In the ABSOLUTE project two tightly interconnected network segments are proposed: an air segment and a terrestrial segment. The former consists of an innovative helium balloon-kite structure carrying LTE aerial Base Stations, while the ground segment consists of Portable Land 
Rapid Deployment Unit complementing network coverage and providing satellite backhaul connectivity. It is important to understand that although LAPs are considered one of the main answers for REN, ground support units are still very essential in complementing such aerial coverage [13], and one of those ground units, is the Relay Node as standardized in LTE-Advanced.

In this paper we are proposing a novel mechanism to enhance the performance of standard relay nodes by utilizing cognitive coverage mechanisms, and hence we are calling those novel relay nodes as Cognitive Relay Nodes (CRN) meant to be rapidly deployable with full self-configuration capabilities, while at the same time, preserving the compatibility with Release $10 \mathrm{RN}$ standard as briefed in Section II. The concept of CRN is outlined in section III, while section IV describes the corresponding network model. Section V presents the proposed cognitive relay assisted emergency network followed by the corresponding simulation results in Section VI. Finally, we provide some concluding remarks in Section VII.

\section{RELAY NODES IN LTE-ADVANCED}

Relaying has been a quite promising trait added to LTE technology, that even before its first standardization in LTEAdvanced Release 10, researchers, academics and vendors were endeavoring to exploit the potential power of this technology that will allow a considerable enhancement in the cell coverage and throughput [8].

Relays are classified into several categories according to their protocol stacking [11]: type Layer-1 is the simplest, that amplifies-and-forward the signal, thus operating only in the physical layer without any packet error correction mechanism, whoever type Layer-2 performs packets decoding and hence is called decode-and-forward, while type Layer-3 has most of eNB features, since it can transmit its own cell identifier, cell specific reference signal (CRS), and primary / secondary reference signals [9], which is also standardized in Release 10 and used hereafter as the base for the enhancements suggested in this paper. In particular Release 10 defines a specific type of Layer-3 called Type- 1 which is an inband relay node having the necessary Radio Resource Control (RRC) functionalities to support cell handover and mobility management, also it has its own scheduler to allocate physical resources to User Equipments (UEs) within its coverage area.

\section{RAPIDLY DEPLOYABLE COGNITIVE RELAY NODE}

The urgent nature of an REN network stipulates a rapid deployable architecture such as network backhaul, eNBs, ground support units, etc... Similarly, our proposed Cognitive Relay Node (CRN) shall allow a swift dispatch via the following features:

- Integration with power supply units such as generator-set, solar panels and backup power.

- Mounted on a wheeled trailer.

- Hydraulic telescopic mast holding the antennas (access and backhaul antennas).

- Auto-tracking directional antenna for backhauling.
- 6-Sectors access antenna with Radio Frequency (RF) beam switching capability.

- Automatic configuration when powered up.

- Selection of donor eNB

- Acquiring Network Parameters.

- Antenna beam control

This paper is focusing on illustrating the enhancement required for the communication system, while "housekeeping" components such as the power system and mechanical structure are not discussed here.

Our proposed CRNs are specifically tailored to complement the coverage of LAP Aerial eNBs (AeNB). During emergency deployments, as shown in Figure 1, the coverage of AeNBs can't be the only source REN service, but rather it should be complemented by terrestrial elements, this fact is due to the limited weight capabilities of LAPs causing limited size of RF power amplifier onboard.

CRNs like any type-1 Relay Node will be backhauled via the standard Un interface to a donor eNB, which is in our case the AeNB). While in its turn AeNB will aggregate all connected CRNs and attached UEs, then forward the traffic to a ground termination point(s).

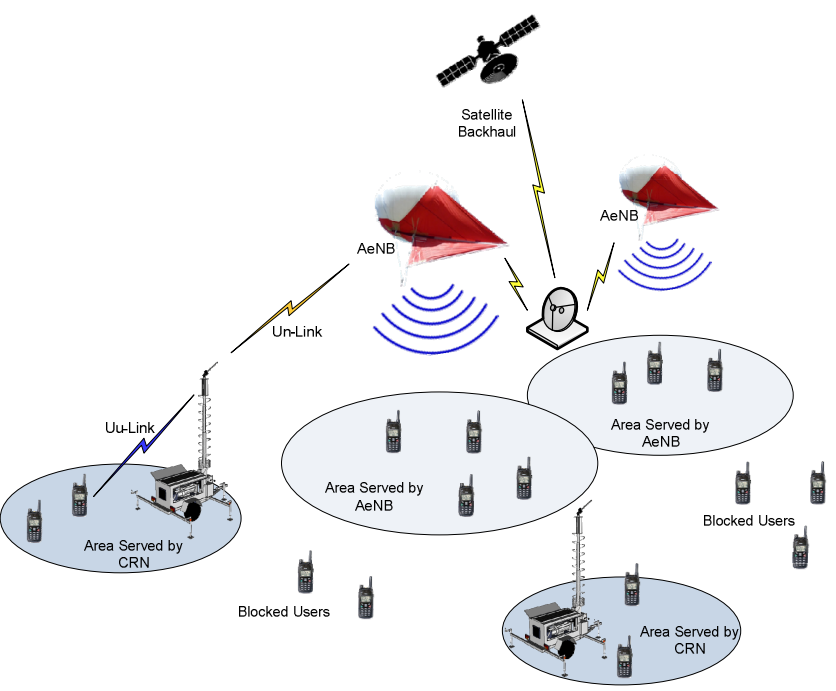

Fig. 1. Overview of REN Architecture with CRNs

\section{IV.NETWORK MODEL}

The model that we have adopted for simulating the performance of our proposed Cognitive Relay Node, aimed to replicate practical scenarios of a Rapid Emergency Network. And accordingly we opt to choose the ABSOLUTE network as our baseline system for the network model.

\section{A. Propagation Model}

The most important part of the wireless network here is the propagation model, this paper consider two models for the propagation as described below:

- Ground-to-Ground RF Propagation (Terrestrial) model.

- Air-to-Ground RF Propagation model.

In the first model we are adhering 3GPP recommendations for the corrected $\mathrm{RN}$ terrestrial propagation in [6] for 
predicting CRN coverage, taking into consideration that RNs has quite different RF propagation behavior than terrestrial eNBs since they are proposed to be mounted on heights of ( 5 to $15 \mathrm{~m}$ ) while models for terrestrial eNBs are typically for heights of (30-100m) [7]. This corrected model assumes two different propagation conditions: (i) Line of Sight (LoS): for UEs in the near proximity with RN and (ii) Non Line of Sight (NLoS): for other UEs. The probability of having a LoS between a Relay Node with a User Equipment of a distance ( $d$ in meters) is strongly depending on the average buildings height and train topography, in our simulation we have chosen to simulate urban environment in consistence with Air-to-Ground propagation model assumptions, that will be discussed shortly. LoS probability in Urban environment is given by [6]:

$$
\mathbb{P}_{L o S}^{R N-U E}(d)=\frac{1}{2}-\min \left(\frac{1}{2}, e^{-\frac{156}{d}}\right)+\min \left(\frac{1}{2}, e^{-\frac{156}{d}}\right)
$$

And accordingly the NLoS probability will be given by:

$$
\mathbb{P}_{N L O S}^{R N-U E}(d)=1-\mathbb{P}_{L O S}^{R N-U E}(d)
$$

In order to combine the two terrestrial propagation scenarios (LoS and NLoS) in a single formula, logarithmic averaging was used, so the resulting path-loss can be written as:

$$
\begin{aligned}
P L^{R N-U E}(d)= & P L_{L O S}^{R N-U E}(d) \cdot \mathbb{P}_{L O S}^{R N-U E}(d) \\
& +P L_{N L O S}^{R N-U E}(d) \cdot \mathbb{P}_{N L O S}^{R N-U E}(d)
\end{aligned}
$$

where $P L_{L o S}^{R N-U E}(d)$ and $P L_{N L O S}^{R N-U E}(d)$ are the LoS and NLoS mean path loss respectively, and are statistically obtained from channel measurement campaigns done in Beijing [6], which resulted the following model for RN height of $5 \mathrm{~m}$ and frequency of 2,000 MHz:

$$
\begin{aligned}
& P L_{L O S}^{R N-U E}(d)=41.1+20.9 \log _{10}(d) \\
& P L_{N L O S}^{R N-U E}(d)=32.9+37.5 \log _{10}(d)
\end{aligned}
$$

On the other hand, the radio coverage serviced by the AeNBs will follow an entirely different scheme. In fact airborne communication has a superior radio propagation ability, compared to conventional terrestrial methods due to the enhanced probability of achieving LoS condition, however technical difficulties prevent deploying large numbers of aerial platforms, and in our baseline network model, we are going to use seven (7) AeNBs only.

For simulating Air-to-Ground propagation we have utilized the profound statistical study developed in [5] that created a standard model for Air-to-Ground path loss, based on three types of paths: (i) Line of sight (LoS) for clear and un-foiled paths, (ii) Obstructed line of sight (OLoS) for partially obstructed paths, and (iii) None Line of Sight (NLoS) for paths that are totally blocked by buildings or other structures.

Similar to ground-to-ground, the combined path loss that consolidates the probability of all types of paths is given by:

$$
\begin{aligned}
& P L^{A e N B-U E}(\theta)=P L_{L o S}^{A e N B-U E}(\theta) \cdot \mathbb{P}_{L O S}^{A e N B-U E}(\theta) \\
& +P L_{O L O S}^{A e N B-U E}(\theta) \cdot \mathbb{P}_{O L O S}^{A e N B-U E}(\theta) \\
& +P L_{N L O S}^{A e N B-U E}(\theta) \cdot \mathbb{P}_{N L O S}^{A e N B-U E}(\theta)
\end{aligned}
$$

where $\theta$ represents the elevation angle between the UE and the serving AeNB and $\mathbb{P}_{L o S}^{e N B-U E}(\theta), \mathbb{P}_{O L O S}^{e N B-U E}(\theta)$, and $\mathbb{P}_{N L O S}^{e N B-U E}(\theta)$ are path probability of LoS, OLoS and NLoS respectively, approximated as per the below path loss formulas:

$$
\begin{aligned}
& P L_{L O S}^{e N B-U E}(\theta)=P L_{0}-20 \log _{10}(\sin \theta) \\
& P L_{O L O S}^{e N B-U E}(\theta)=P L_{0}+4.77+0.35 e^{\frac{(90-\theta)}{21.04}} \\
& P L_{N L O S}^{e N B-U E}(\theta)=P L_{0}+15.15-12.62 e^{\frac{(90-\theta)}{-7.32}}
\end{aligned}
$$

where $P L_{0}$ is the free space loss [12] between the AeNB and the ground point directly beneath the LAP:

$$
P L_{0}=20 \log \left(h_{L A P}\right)+38.47
$$

In ABSOLUTE network each aerial platform is a sectorized eNB carrying three directional antennas, and in our network model we chose to orient them towards the cells' centers.

So the down tilt of all antennas will be given by:

$$
\theta_{\text {tilt }}=\tan ^{-1}\left(\frac{h_{L A P}}{R_{\text {cell }}}\right)
$$

where $h_{L A P}$ denotes the average LAP altitude (meters), and $R_{\text {cell }}$ is the design cell radius (meters), which is represented by the cell's hexagon radius, as shown in Figure 2.

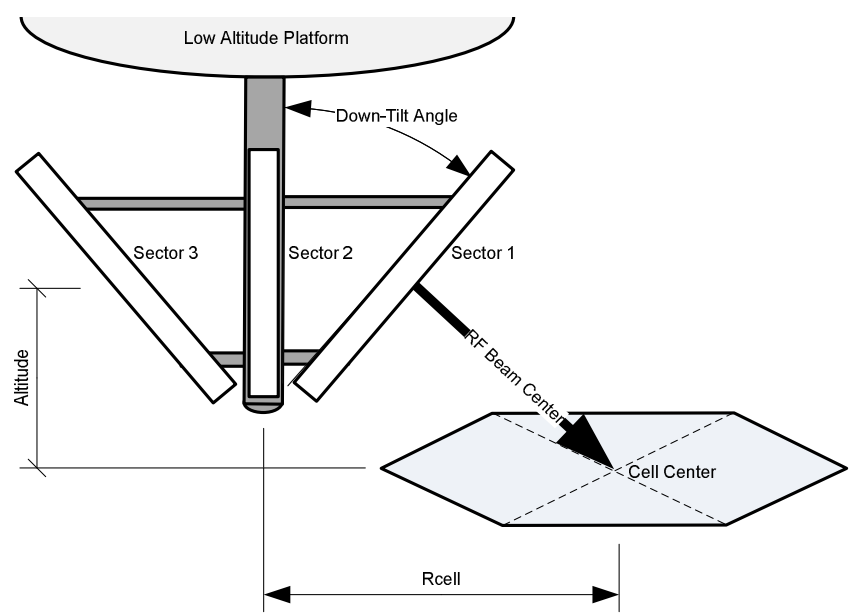

Fig. 2. The proposed AeNB Antenna configuration, adopting the requirements from the ABSOLUTE project [2].

\section{COGNITIVE RELAy AsSisted EMERGENCY NETWORK}

As stated previously, the Cognitive Relay Nodes are aimed to assist the coverage served by the aerial base stations (AeNB), and as an example, Figure 3, shows a target square 
area where REN coverage is needed. The three AeNBs are providing the main service; while the $9 \mathrm{CRNs}$ are working in a cognitive manner (by steering their RF beams) for serving the coverage gaps left by the AeNBs.

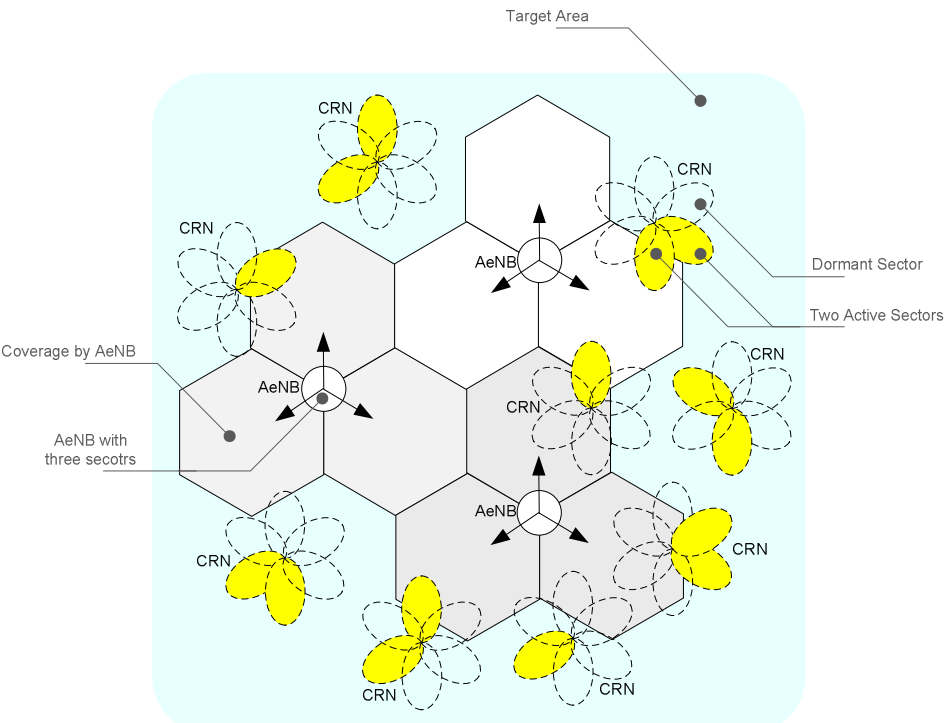

Fig. 3. Illustrating the concept of CRN Assisted REN

So the intelligence in our proposed CRN is mainly achieved by adopting three mechanisms (i) the ability to shutdown the transmitting power when no users are spotted in the CRN's proximity, which will allow an enormous power saving, (ii) the ability to Calculate Pathloss with UEs before commencing the relaying TX coverage, (iii) CRNs will be fit with switched antenna set that will allow directing RF power towards the UEs that are in most need for the service.

These three mechanisms will be further elaborated in the following sections.

\section{A. Cognitive Relaying Mechanism}

After powering up the CRN, it will acquire its location via a positioning system, such as GPS/Galileo, while at the same time, it will temporary attach to the strongest available AeNB, and acquire the list of all LAPs current coordinates / heights (via a high level application), then the CRN will mechanically steer its backhaul antenna towards the nearest and less-congested AeNB and re-attach. Refer to Figure 4 showing the operation sequence. After that the CRN will start rotating the beam of the access antenna (electronically) several times per second in order to construct users profile list, upon that the decision on which sector(s) to lock the access beam will be taken.

For each transceiver the CRN will continuously assess the UEs conditions of neighboring sectors, and accordingly the UE profile will be maintained for three consecutive sectors only per transceiver, i.e. the served sector and the two adjacent ones. If there is no more nominated UEs to be served, the transmitter(s) of the CRN will switch off the TX radio power and enter into a dormant mode, in which the CRN will keep rotating the access beam (in receiving mode only) and try to construct the table of any approaching user(s). CRN will have the capability of steering radio beams towards $\mathrm{UE}(\mathrm{s})$ that are in most need of the service. In contrary to conventional Relay Nodes (in nominal networks) that are operator-deployed and well planned to serve certain cell-edge locations, also designed to have Omni directional transmission, in REN the ability to perform full RF planning will be very limited since there is neither enough time nor enough information about the afflicted area, accordingly the deployment of intelligent self-configure RNs will be the key to REN success.

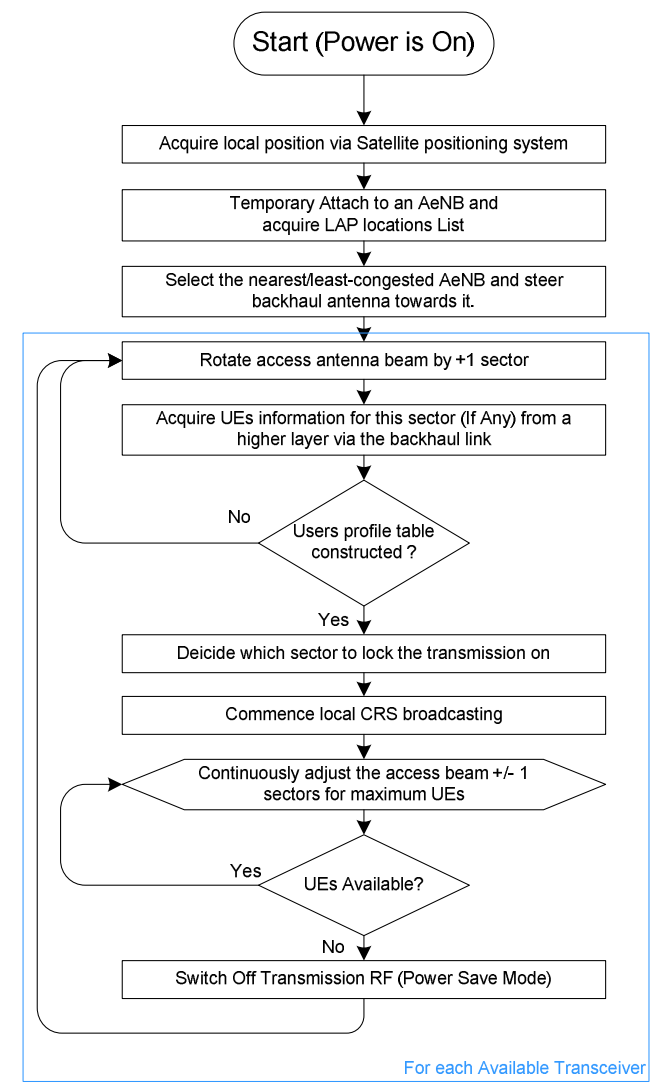

Fig. 4. The process of CRN intelligently attaching to an AeNB.

\section{B. RN-UE Path Loss Predication}

A key elementary feature in LTE System is the ChannelState Reporting [1] provided by the User Equipments in order to allow eNBs to perform channel depended scheduling and the Adaptive Modulation and Coding (AMC), one portion of this report is called the Channelquality indicator (CQI) representing the highest modulation scheme that the downlink channel transmission can take place with a block-error rate of at most $10 \%$. CRN will utilize both $C Q I$ feedback and power reports received by the AeNB in order to perform decisions on where to steer the access antenna RF beam. A Path Loss prediction for the RNUE path will be accomplished via the following procedure: CRN will listen to UE (number $\mathrm{n}$ ) or $U E_{n}$ transmitting the Physical Uplink Control Channel (PUCCH) that will be received by the $\mathrm{CRN}$ according to the following:

$$
P_{U E_{n}}^{R X, C R N}=P_{U E_{n}}^{T X}-P L_{U E_{n}-C R N}
$$


where $P_{U E n}^{T X}$ is the power transmitted by the $U E_{n}$, and $P L_{U E-C R N}$ is the Pathloss between $U E_{n}$ and CRN. On the other hand $P_{U E n}^{T X}$ can be predicted by the AeNB since it follows a well-know LTE specific formula:

$$
P_{U E_{n}}^{T X}=\min \left\{P_{\text {max }}, P_{0, P U C C H}+P L_{D L}+\Delta_{\text {format }}+\delta\right\}
$$

where $P_{\text {max }}$ is the maximum allowable transmit power and $P_{0, P U C C H}$ is a cell specific parameter, $\Delta_{\text {format }}$ is a format dependent offset and $\delta$ is a power control offset. What is important to distinguish is that all above mentioned parameters are well known by the AeNB except the downlink path loss $P L_{D L}$, accordingly the formula can rewritten as:

$$
P_{U E_{n}}^{T X}=f\left(P L_{D L}\right)
$$

On the other hand, the received power at the AeNB will be given by:

$$
P_{U E_{n}}^{R X, e N B}=P_{U E_{n}}^{T X}-P L_{U L}
$$

where $P L_{U L}$ is the uplink path loss that could be considered equal to $P L_{D L}$, i.e. reciprocal channel. From (14) and (15) the AeNB can predict the transmitted power by the user equipment $P_{U E_{n}}^{T X}$ and then report this power along with CQI to the CRN, that will then use (12) to obtain $U E_{n}-C R N$ path loss estimation.

\section{Switched Beam Antenna}

As mentioned previously, the access link is equipped with an electronically switched beam antenna capable of steering the RF power to/from a certain direction (for each transceiver available in the CRN), a mechanism in which the CRN will be able to serve the UEs that are in most need of the service. Also, directing $\mathrm{CRN}$ beam will mitigate the interference on AeNB coverage since the frequency reuse factor in LTE networks is unity, and interference control is a key for spectrum utilization efficiency.

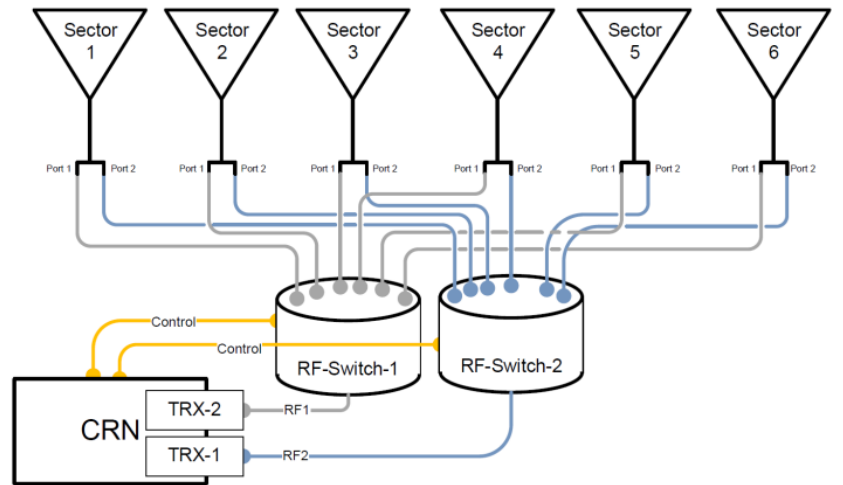

Fig. 5. Switched Antenna in CRN (With two Transceivers)

In order to enable this mechanism 6-directional antennas are connected to RF switches as illustrated in Figure 5 (showing a CRN with two transceivers configuration example), these RF switches are electrically-controlled by the CRN providing steering of RF coverage towards the desired direction(s), the only minor negative impact of introducing the RF switch through the antenna line is its insertion loss (which is in the range of $0.8 \mathrm{~dB}$ ), however , as it will be shown in the simulation, that the overall coverage enhancement will overcome this minor impact.

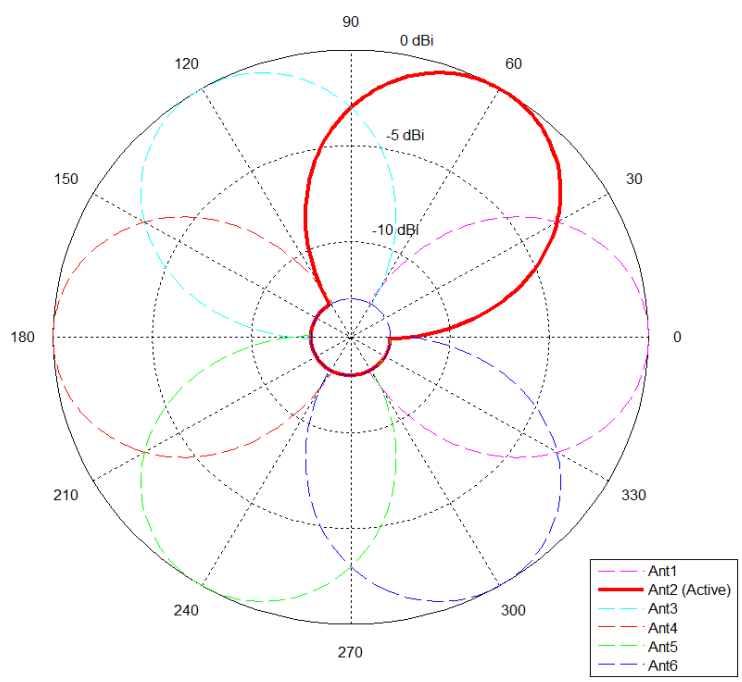

Fig. 6. Beam Steering for directing RF power in the network

Each antenna is assumed to have 65 degrees of half power beam width in order to allow sufficient overlapping between sectors. It is important to note that a $\mathrm{CRN}$ might have more than one set of transceiver/antennas and the number of the simultaneous active sectors will be equal to the available transceiver/antenna sets. The radio power of a certain transceiver will be directed towards a single direction, and thus having a superior RF performance in this direction of interest. Figure 6 illustrate this concept, one active sector is shown in red solid line.

\section{Beam Steering Decision}

So far, the mechanisms of how to perform UE-RN path loss prediction have been illustrated and also how to steer the RF beam, while this section will show the method of deciding which sector(s) to serve based on the these mechanisms.

In fact, during beam rotation operation period, the CRN is proposed to build a User Equipment Table that has the following general format:

TABLE I

UE Database formed by the CRN

\begin{tabular}{cccc}
\hline \hline $\begin{array}{c}\text { Sector } \\
\text { Number }\end{array}$ & UE ID & $\begin{array}{c}\text { UE-CRN } \\
\text { Path Loss }\end{array}$ & CQI \\
\hline Sector 1 & $\mathrm{UE}_{1}$ & $\mathrm{PL}_{1}$ & $\mathrm{CQI}_{1}$ \\
Sector 1 & $\mathrm{UE}_{2}$ & $\mathrm{PL}_{2}$ & $\mathrm{CQI}_{2}$ \\
$\cdot$ & $\cdot$ & $\cdot$ & $\cdot$ \\
Sector 2 & $\mathrm{UE}_{15}$ & $\mathrm{PL}_{15}$ & $\mathrm{CQI}_{15}$ \\
$\cdot$ & $\cdot$ & $\cdot$ & $\cdot$ \\
$\cdot$ & $\cdot$ & $\cdot$ & $\cdot$ \\
Sector 6 & $\mathrm{UE}_{40}$ & $\mathrm{PL}_{40}$ & $\mathrm{CQI}_{40}$ \\
\hline \hline
\end{tabular}

So for each sector a complete list of detectable UEs will be constructed, and then the sector will be given a score value according to the following formula: 


$$
S_{i}=\sum_{n_{o}}^{N}\left(W_{1} \cdot \frac{1}{P L_{U E_{n}-C R N}}+W_{2} \cdot \frac{1}{C Q I_{n}}\right)
$$

where $\mathrm{W}_{1}$ and $\mathrm{W}_{2}$ are tuning weights, and $C Q I_{n}$ is the average CQI reported by a user-equipment $U E_{n}$ to the serving AeNB, and $\mathrm{N}$ is the total number of detectable UEs within the sector. Basically, the score of each UE is inversely proportional to its predicted path loss and to its reported CQI. After completing sectors scoring, the CRN will lock the RF switch to the sector(s) with the highest score, and commence the cell broadcasting. For example in the case of two-transceivers the $\mathrm{CRN}$ will be able to activate two sectors as a maximum.

\section{E. Relay Nodes Placement Strategy}

During REN deployment, agencies have very limited time for performing radio network planning and setup, accordingly an assumed planning tool shall nominate the relay nodes locations based on the number of deployed LAPs and the terrain average parameters. In our network model we have used a CRN location planning method based on the spatial blockage probability; first of all we obtain AeNBs coverage simulation for the target area (without the effect of any $\mathrm{CRN}$ ), then we break down obtained coverage simulation map to smaller adjacent circles of a radius equal to the design cell radius of the CRN, after that we calculate the spatial service blockage probability inside each of those circles by counting the blocked pixels:

$$
\mathbb{P}_{\mathrm{RN}_{\mathrm{i}}}^{\text {Spatial }}=\frac{\mathrm{N}_{\text {blocked }}}{\mathrm{N}_{\text {total }}}
$$

where $\mathrm{N}_{\text {blocked }}$ is the number of pixels that are not serviced (blocked), and $\mathrm{N}_{\text {total }}$ is the total number of simulated pixels within the study circle, as depicted in Figure 7.

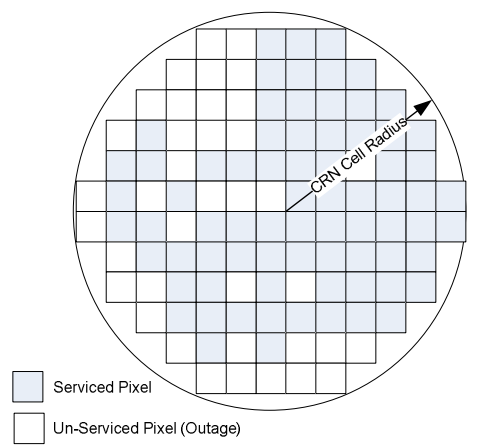

Fig. 7. Illustrating the concept of geographical pixels service by AeNB.

A pixel is considered as blocked, when it has an SINR less than the service threshold SINR required for attaining certain service throughput. Thus a decision whether to place a $\mathrm{CRN}_{\mathrm{i}}$ or not in the center of this study circle is given by the following Boolean condition:

$$
\text { Deploy } R N_{i}= \begin{cases}0, & \mathbb{P}_{R N_{i}}^{\text {Spatial }}>S B_{t h} \\ 1, & \mathbb{P}_{R N_{i}}^{\text {Spatial }} \leq S B_{t h}\end{cases}
$$

where $\mathrm{SB}_{t h}$ is the Spatial Blockage decision threshold and is related to the overall target network quality of service (QoS). It is important to notice that this method is entirely different than the commonly studied RN deployment schemes for terrestrial nominal networks [10].

\section{SIMULATION ANALYSIS}

The assumed baseline REN Network consists of seven (7) Low Altitude Platforms covering a square geographical area of $40 \mathrm{~km} \times 40 \mathrm{~km}$, replicating an urban environment of a typical European city, with an assumed average building height of $11.4 \mathrm{~m}$ and flat average ground level [5], the optimum altitude of the platforms was obtained by searching the maximum service availability ratio among several simulation runs of different LAP altitudes. The results in Figure 8 are showing a peak service at around 1,650 m. This altitude is mainly dependent on the average building height, transmission power and on the target SINR. Generally, higher buildings demands higher LAP altitude.

Two factors are affecting the produced curve in Figure 8, first the enhancement of coverage when LAP altitude is rising between $0 \mathrm{~m}$ and $1,650 \mathrm{~m}$, after that comes the effect of path loss factor, starting to be more significant, and negatively affecting the service.

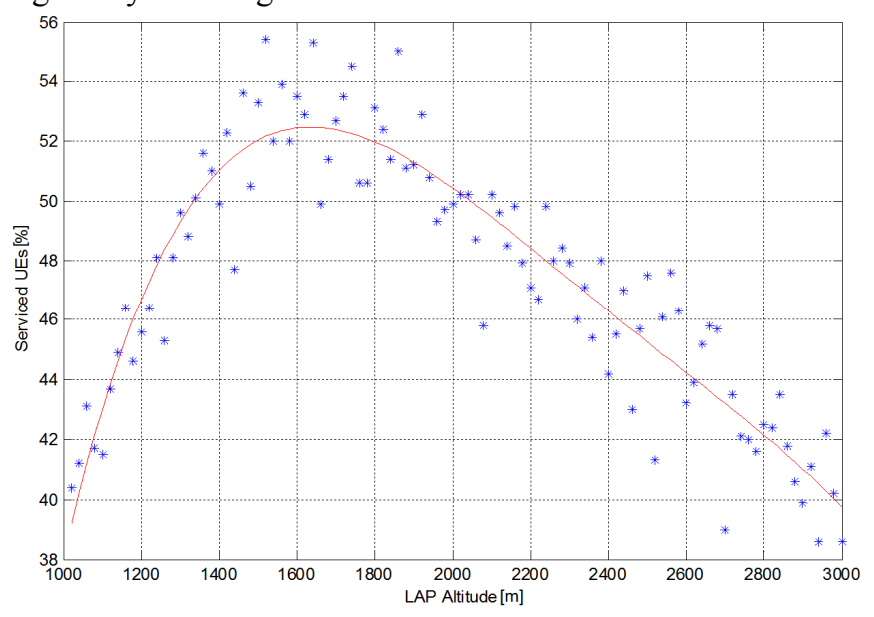

Fig. 8. Simulation results for obtaining the Optimum LAP Altitude for the assumed urban environment.

\section{A. Simulation Scenarios}

In order to illustrate network performance impact of introducing the cognitive relay nodes three different scenarios have been simulated, Scenario (1) represents our baseline, and consists of the aerial eNodeBs only, without any terrestrial relaying capabilities, while in Scenario (2) conventional Type-1 Relay Nodes were added in order to assist the coverage of the AeNBs. It is important to notice here that the proposed conventional RNs has the same radio transmitting power as of the CRNs, but instead of the switched antennas system, standard omni-directional antennas are fitted.

In Scenario (3), conventional RNs are replaced with our Cognitive Relay Nodes for complementing the aerial coverage. Table-II summarizes the main system parameters used in the simulation of our network and scenarios. Users are assumed to be randomly distributed within the service 
area which is a circle of radius $20 \mathrm{~km}$, having a total number of 2,000 UEs.

TABLE II

SIMULATION PARAMETERS

\begin{tabular}{|c|c|}
\hline Parameter & Value \\
\hline \multirow[t]{2}{*}{ Cellular Layout } & $\begin{array}{l}\text { For AeNBs: Hexagonal grid } \\
3 \text { sectors per site } 0^{\circ} \quad 120^{\circ} 240^{\circ}\end{array}$ \\
\hline & $\begin{array}{l}\text { For CRNs: } \\
6 \text { Secor per site } 0^{\circ} 60^{\circ} 120^{\circ} 180^{\circ} \\
240^{\circ} 300^{\circ} \\
\text { Up to Two active sectors at a time }\end{array}$ \\
\hline Propagation Environment & Urban (City) \\
\hline Simulation Frequency & $2,000 \mathrm{MHz}$ \\
\hline System Bandwidth & $5 \mathrm{MHz}$ \\
\hline AeNB Antenna Max Gain & $8 \mathrm{dBi}$ [ABSOLUTE Project] \\
\hline AeNB Antenna H-Beam Width & $60^{\circ}$ degrees [ABSOLUTE Project] \\
\hline AeNB Antenna V-Beam Width & $60^{\circ}$ degrees [ABSOLUTE Project] \\
\hline AeNB Antenna Port power & $23 \mathrm{dBm}$ [ABSOLUTE Project] \\
\hline LAP Height (AeNB) & $1,650 \mathrm{~m}$ \\
\hline CRN Antenna Max Gain & $18 \mathrm{dBi}$ \\
\hline CRN Antenna H-Beam Width & $65^{\circ}$ \\
\hline CRN Antenna V-Beam Width & Effect Not Simulated \\
\hline CRN Antenna Height & $5 \mathrm{~m}$ \\
\hline $\begin{array}{l}\text { CRN Antenna Port power (Minus } \\
\text { RF switch Loss and } \\
\text { Cables/Connectors loss) }\end{array}$ & $37.7 \mathrm{dBm}$ \\
\hline $\begin{array}{l}\text { Conventional RN Antenna Max } \\
\text { Gain }\end{array}$ & 6 dBi (Omni Directional) \\
\hline $\begin{array}{l}\text { Conventional RN Antenna H- } \\
\text { Beam Width }\end{array}$ & $360^{\circ}$ \\
\hline $\begin{array}{l}\text { Conventional RN Antenna V- } \\
\text { Beam Width }\end{array}$ & Effect Not Simulated \\
\hline Conventional RN Antenna Height & $5 \mathrm{~m}$ \\
\hline $\begin{array}{l}\text { Conventional RN Antenna Port } \\
\text { power (Minus Cables/Connectors } \\
\text { loss) }\end{array}$ & $38.5 \mathrm{dBm}$ \\
\hline UE Antenna gain & $0 \mathrm{dBi}$ \\
\hline UE Noise Figure & $9 \mathrm{~dB}$ \\
\hline
\end{tabular}

\section{B. Simulation Results}

The performed simulation mainly focuses on obtaining the achieved RSRP (Reference Signal Received Power) for each of the explained scenarios, because RSRP is the main coverage indicator of how well the target area is being served. RSRP result for the first simulation scenario is depicted in Figure 9, that also shows the locations of the assumed seven (7) AeNBs (the red dots) and their hexagon cellular structure. Areas with SINR below the service threshold level are colored in white indicating service outage. It is noticeable that a considerable portion of the target area is outside the coverage service.

While Figure 10 (scenario 2) depicts the RSRP results of the combined coverage of AeNBs and conventional relay nodes with omni-directional antennas.

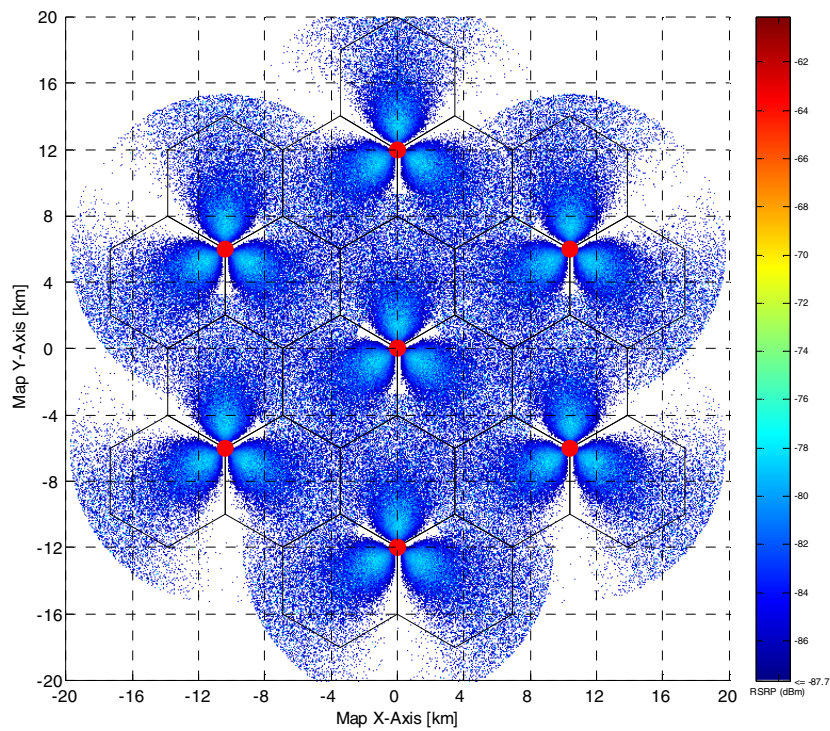

Fig. 9. RSRP Scenario 1 (AeNBs Only)

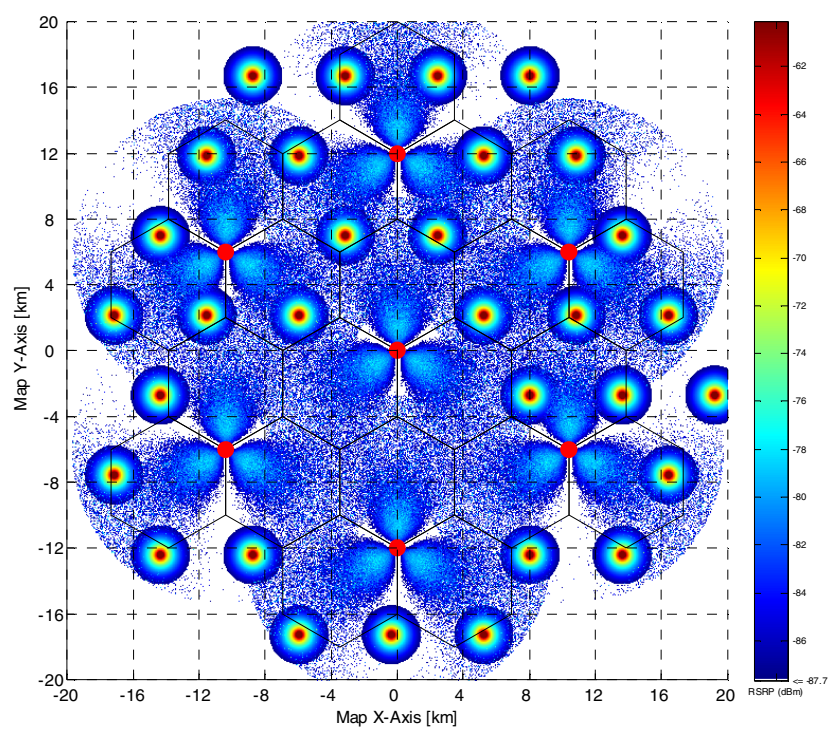

Fig. 10. RSRP Scenario 2 (AeNBs and Conventional RNs)



Fig. 11. RSRP Scenario 3 (AeNBs and CRNs) 
The situation in scenario (3) is showing a significant enhancement of the service, with the introduction of CRNs. The placement of CRNs was done according to the planning method described earlier that yielded 32 CRNs.

Figure 11 depicts the resulting combined coverage of AeNBs and CRNs. It can be noticed from the figure that most of the CRNs activated both sectors, covering the service gaps left by the aerial network. In order to present more quantitative indication of the gained enhancement, a cumulative distribution function is plotted comparing the SINR of the three simulated scenarios (Figure 12).

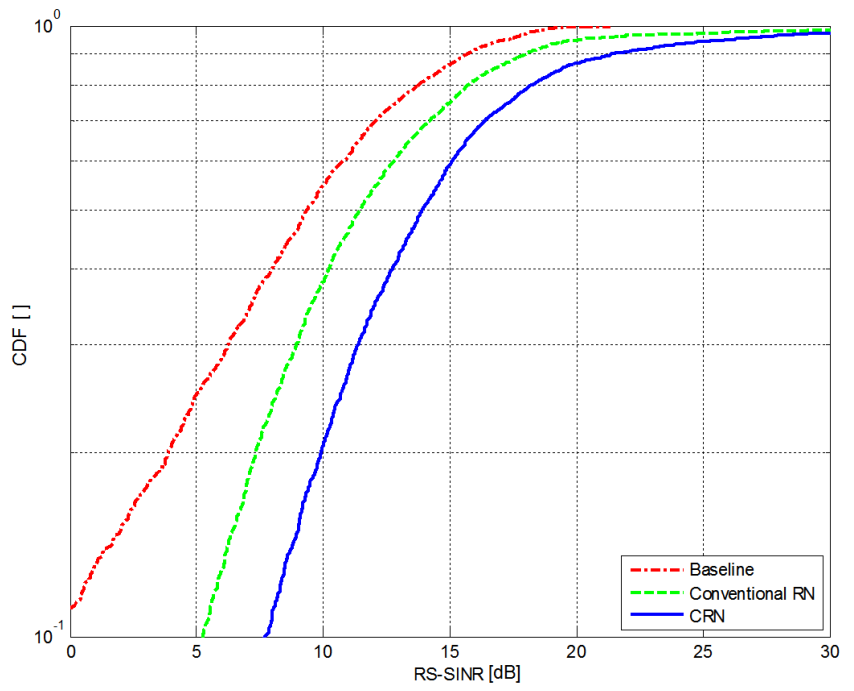

Fig. 12. Comparative CDF Plot for the three Scenarios

Finally Figure 13 shows a comparison between the service blockage probabilities of the target systems calculated based on all assumed UEs in the model.

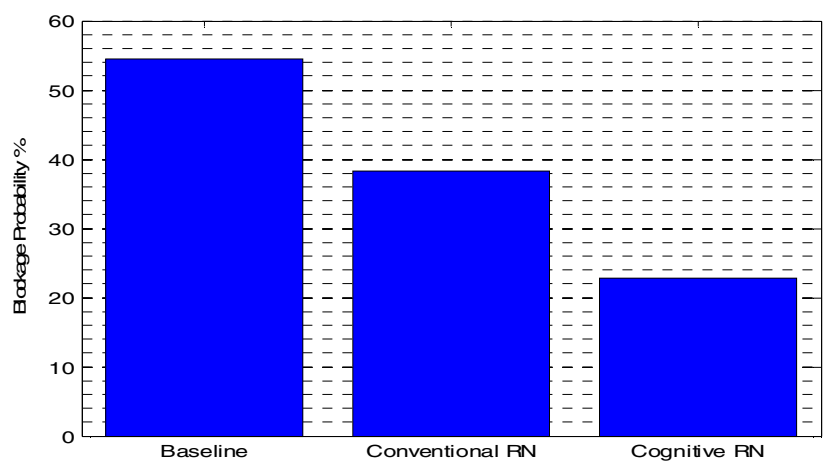

Fig. 13. Comparison between the service blockage probability.

\section{VII.CONCLUSION}

This paper has introduced a novel practical approach in realizing cognitive relay nodes for serving rapidly deployable emergency networks. The obtained results show significant performance enhancement over conventional relay nodes in terms of service and coverage. Although the simulation was conducted for a specific REN architecture, $\mathrm{CRN}$ concept can be adopted in some special scenarios related to commercial networks. Our future work in this field might include the extension of this study to various propagation models as well as the experimental verification of the CRN concept.

\section{ACKNOWLEDGMENT}

The research was partially funded by the ABSOLUTE project from the European Commission's Seventh Framework Programme (FP7-2011-8) under the Grant Agreement FP7-ICT-318632 [2].

\section{REFERENCES}

[1] E. Dahlman, S. Parkvall, J. Skold "LTE/LTE-Advanced for Mobile Broadband" Academic Press, PP282-287.

[2] EU-FP7 IP Project ABSOLUTE: URL - http://www.absolute-project.eu/

[3] Federal Communications Commission "The Role of Deployable Aerial Communications Architecture in Emergency Communications and Recommended Next Steps" 2011.

[4] Alcatel Lucent, Government Technology "How-to Guide: for LTE in Public Safety" Government Technology Magazine, 2010.

[5] F. Qixing, J. McGeehan, E. K. Tameh, and A. R. Nix, "Path Loss Models for Air-to-Ground Radio Channels in Urban Environments," in VTC, 2006. VTC. IEEE 63rd, 2006, pp. 2901-2905.

[6] 3GPP R1-091566 " Relay to UE channel model for LTEAdvanced", China Mobile, Vodafone, 2009.

[7] G. Stuber, " Principles of Mobil Communication" published by Springer, 2011, Chapter 2.

[8] Yifei Yuan "LTE-Advanced Relay Technology and Standardization", Chapter1, published by Springer, 2013.

[9] ARTIST 4G "Enhancements to Type-1 Relay Implementation", URL: ict-artist4g.eu, 2012.

[10] 3GPP TSG-RAN WG1 Meeting \#60 " Type-1 Relay Performance for Uplink"2010.

[11] M. Iwamura, H. Takahashi, S.Nagata "Relay Tecnhnology in LTE-Advanced", NTT DOCOMO Technical Journal Vol.12 No.2.

[12] A. Raisanen, A. Lehto "Radio Engineering for Wireless Communication and Sensor Applications" Published by Artech House, pp304-5.

[13] A. Hourani and S. Kandeepan "Temporary Cognitive Femtocell Network for Public Safety LTE", IEEE CAMAD 2013, Berlin.

[14] S. Kandeepan and A. Giorgetti, Cognitive Radio Techniques: Spectrum Sensing, Interference Mitigation and Localization, Artech House, London, 2013.

[15] M. Mueck et. al, ETSI Reconfigurable Radio Systems Status and Future Directions on Software Defined Radio and Cognitive Radio Standards IEEE Communications Magazine, Sep 2010.

[16] S. Kandeepan, A. Giorgetti and M. Chiani, Periodic Spectrum Sensing Performance and Requirements for Legacy Users with Temporal and noise Statistics in Cognitive Radios, GLOBECOM Workshops IEEE, pp. 1-4, Dec. 2009.

[17] S. Kandeepan, R. Piesiewicz, T. Aysal, A. Biswas and I. Chlamtac, Spectrum Sensing for Cognitive Radios with Transmission Statistics: Considering Linear Frequency Sweeping, EURASIP Journal or Wireless and Communication Networks, Vol. 2010, Jan 2010 Art No. 6. 
[18] S. Kandeepan, L. Nardis, M. G. Benedetto, G. Corazza, Alessandro, Cognitive Satellite Terrestrial Radios, IEEE Globecom, Dec 2010, Florida.

[19] S. Kandeepan et. al, Experimentally Detecting IEEE 802.11n Wi-Fi Based on Cyclostationarity Features for Ultra-Wide Band Cognitive Radios, IEEE PIMRC 2009, Tokyo.

[20] S. Kandeepan, et. al, Time-Divisional and Time-Frequency Divisional Cooperative Spectrum Sensing, IEEE Crowncom, Hannover, June 2009.

[21] T. Aysal, Kandeepan. S, Radoslow. P, Cooperative Spectrum Sensing over Imperfect Channels, IEEE BWAWS, Globecom, 2008

[22] T. Aysal, Kandeepan. S, Radoslaw. P, Cooperative Spectrum Sensing with Noisy Hard Decision Transmissions, International Conference on Communications (ICC), 14-18 June , Dresden 2009

[23] A. Rahim, T. Aysal, Kandeepan. S, Dzmitry. K, Radoslaw. P, Cooperative Shared Spectrum Sensing for Dynamic Cognitive Radio Network, International Conference on Communications (ICC), 14-18 June, Dresden 2009

[24] S. Kandeepan et. al, Periodic Sensing in Cognitive Radios for Detecting UMTS/HSDPA Based on Experimental Spectral Occupancy Statistics, IEEE WCNC, April 2010

[25] S. Kandeepan et. al, Distributed ring-around Spectrum Sensing for Cognitive Radio Networks, IEEE ICC 2011, Kyoto

[26] S. Kandeepan, et. al, Preliminary Experimental Results on the Spectrum Sensing Performances for UWB-Cognitive Radios for Detecting IEEE 802.11n Wi-Fi Systems, IEEE ISWCS, Sienna, Sep 2009

[27] S. Kandeepan et. al, Spectrum Sensing for Cognitive Radios with Transmission Statistics: Considering Linear Frequency Sweeping, JWCN EURASIP, April 2010.

[28] S. Kandeepan, et al, Bayesian Tracking in Cooperative Localization for Cognitive Radio Networks, IEEE VTC, 2629 April, Barcelona 2009.

[29] A. Mariani, S. Kandeepan, A. Giorgetti and M. Chiani, Cooperative Weighted Centroid Localization for Cognitive Radio Networks, IEEE ISCIT, 2-5 October, 2012, Gold Coast.

[30] S.Arunthavanathan, S. Kandeepan, R.J. Evans, 'Spectrum Sensing and Detection of Incumbent-UEs in Secondary-LTE based Aerial-Terrestrial Networks for Disaster Recovery',IEEE CAMAD, Sept 2013, Berlin. 